\title{
COMUNICACIÓN
}

\section{Fasciolosis en equinos fina sangre de carrera de los hipódromos de la zona central de Chile. 2002-2003}

\author{
HÉCTOR ALCAÍNO*, LEOPOLDO PARRA* y TEXIA R. GORMAN*
}

\section{THOROUGHBRED RACEHORSES FASCIOLOSIS IN RACETRACKS OF THE CENTRAL ZONE OF CHILE}

The purpose of this survey is to add more information about fasciolosis in thoroughbred racehorses located at the racetracks of the Metropolitan (Hipódromo Chile de Santiago (HCH) and Club Hipico de Santiago (CHS)) and the V Region (Valparaiso Sporting Club de Viña del Mar (VSC)) of Chile and, indirectly, to evaluate how sanitary plans have been working in the recent years. Horses faecal samples were collected between september 2002 and june 2003. These samples were obtained from 666 thoroughbred racehorses; the number of samples was proportional to total population of racehorses in each racetracks during the collection period. $(\mathrm{HCH}=236 ; \mathrm{CHS}=306$ and $V S C=124$ samples). A $6 \%$ of racehorses were positive for the presence of Fasciola hepatica eggs in the faecal sedimentation test. Positive horses were only found at both Metropolitan Region. racetracks (HCH 6,8\% and CHS 8,5\%). No differences were observed with regard to age and sex of the animals. $(p>0,05)$. The prevalence of infection has decreased in the last 20 years $(p<0,05)$.

Key words: Fasciola hepatica, Fasciolosis, Horse diseases, Survey, Chile.

\section{INTRODUCCIÓN}

El principal objetivo que se persigue con los caballos Fina Sangre de Carrera (F.S.C.), es obtener un máximo rendimiento en las pistas de carrera. Para lograr este propósito es importante mantener al animal en un óptimo estado de salud. Dentro de las patologías que pueden afectar el rendimiento de los caballos están las enfermedades parasitarias y dentro de ellas las infecciones producidas por el tremátodo Fasciola hepatica. Aquellos equinos infectados con este parásito presentan diversos signos clínicos como decaimiento, anorexia, diarrea, cólicos digestivos, ictericia, y bajo rendimiento en las actividades deportivas junto a una progresiva pérdida de su condición corporal ${ }^{1-3}$.

Esta comunicación tendrá como objetivo primordial actualizar la información sobre la fasciolosis de los equinos F.S.C. en "training" de los hipódromos centrales de Chile. Este estudio además de complementar los trabajos previos ${ }^{4-}$ ${ }^{10}$, permitirá determinar si ellos tuvieron algún efecto en despertar la inquietud por el control del parásito por parte de los propietarios y/o médicos veterinarios.

\section{MATERIAL Y MÉTODOS}

Desde septiembre del año 2002 a junio del

\footnotetext{
* Departamento de Medicina Preventiva Animal. Facultad de Ciencias Veterinarias y Pecuarias. Universidad de
} Chile. Casilla 2 Correo 15, La Granja, Santiago, Chile. halcaíno@uchile.cl 
2003, se obtuvieron muestras de heces de 666 caballos F.S.C de diferentes corrales de los 3 hipódromos centrales de Chile. Este número de muestras fue determinado mediante una fórmula para estudios descriptivos de poblaciones ${ }^{11}$. considerando un nivel de confianza de $95 \%$, una prevalencia hipotética de 10,44\% (basada en un estudio realizado con anterioridad $)^{7}$ y un margen de error de $20 \%$.

La población total de equinos F.S.C residentes en los 3 hipódromos fue de 3.500 individuos; lo que se descompone en 1.242 caballos $(35,5 \%$ de la población total) residentes en el Hipódromo Chile de Santiago (HCH), 1.612 caballos $(46 \%)$ residentes en el Club Hípico de Santiago (CHS) y 650 caballos $(18,5 \%)$ residentes en el Valparaíso Sporting Club de Viña del Mar (VSC). Todo esto implica que la obtención de las 666 muestras de heces se debió distribuir en la misma proporción (236 del HCH, 306 del CHS y 124 del VSC).

Con este mismo criterio de proporciones se distribuyó el número de muestras a obtener desde cada corral (esto significó muestrear aproximadamente el 19\% de los caballos disponibles de cada corral). Los F.S.C a los que se les colectaron heces, fueron seleccionados al azar (cada 5 ejemplares) a partir de la segunda pesebrera situada a la derecha de la entrada de cada corral; de tal manera que se extrajeron heces de los equinos ubicados en las pesebreras $\mathrm{N}^{\circ} 2,7,12$, 17, 22, etc.

Posteriormente, en el laboratorio, se realizó un examen coprológico de sedimentación a $10 \mathrm{~g}$ de cada muestra de excremento, en búsqueda de huevos de $F$. hepatica ${ }^{12}$. Los resultados obtenidos en este estudio se compararán mediante la prueba de chi cuadrado $(\mathrm{p}<0,05)$ con los que se obtuvieron hace 20 años en los mismos hipódromos ${ }^{7}$.

\section{RESULTADOS}

Se encontró la presencia de huevos de $F$. hepatica en las heces de 40 de los 666 F.S.C examinados, lo que significa una prevalencia del $6 \%$. Los resultados obtenidos según procedencia de los caballos (Hipódromos de Santiago ( $\mathrm{HCH}$ y CHS) o de Viña del Mar (VSC) ) se presentan en la Tabla 1. Las diferencias que se observan entre los resultados obtenidos en los equinos F.S.C. radicados en los hipódromos de Santiago con aquéllos obtenidos en el V.S.C., indicaron diferencias significativas a la prueba de $\chi^{2}(\mathrm{p}<$ $0,05)$.

En la Tabla 2 se expone la distribución de las prevalencias de infección por $F$. hepatica según hipódromos en donde estaban radicados los equinos F.S.C. al momento de realizarse el estudio. No hubo diferencias significativas entre las prevalencias obtenidas de los dos hipódromos de la ciudad de Santiago ( $p>0,05)$, pero si hubo diferencias entre las prevalencias de cada hipódromo de Santiago con la observada en el Valparaíso Sporting Club de Viña del Mar. ( $\mathrm{p}<$ $0,05)$.

La distribución de la infección según sexo y edad se muestran en la Tablas 3 y 4 . No hubo diferencias significativas según ambas variables. $(\mathrm{p}>0,05)$.

En la Tabla 5 se presentan los resultados obtenidos en el estudio realizado en el año $1983^{7}$ y los obtenidos en el presente estudio. La diferencia de infección global obtenida en el presente estudio en los tres hipódromos (6\%, 40/666) con respecto a los resultados obtenido hace 20 años atrás $(10,4 \%, 111 / 1.063)$, fue significativas $(\mathrm{p}<0,05)$. Lo mismo sucedió con las diferencias obtenidas en cada uno de los hipódromos $(\mathrm{p}<0,05)$.

Tabla 1. Prevalencia de infección por Fasciola hepatica en caballos F.S.C. de los hipódromos centrales de Chile y su distribución según ciudades

\begin{tabular}{lccc}
\hline Ciudad & $\begin{array}{c}\text { Caballos } \\
\text { F.S.C } \\
\text { Examinados }\end{array}$ & \multicolumn{2}{c}{$\begin{array}{c}\text { Infectados con } \\
\text { Fasciola hepatica } \\
\text { n }\end{array}$} \\
\hline Santiago & 542 & 40 & 7,4 \\
Viña del Mar & 124 & 0 & 0,0 \\
Total & 666 & 40 & 6,0 \\
\hline
\end{tabular}

Tabla 2. Prevalencia de infección por Fasciola hepatica en equinos F.S.C. en los hipódromos centrales de Chile

\begin{tabular}{lcrc}
\hline Hipódromo & $\begin{array}{c}\text { Caballos } \\
\text { F.S.C } \\
\text { Examinados }\end{array}$ & $\begin{array}{c}\text { Infectados con } \\
\text { Fasciola } \\
\text { n }\end{array}$ & $\begin{array}{c}\text { hepatica } \\
\text { \% }\end{array}$ \\
\hline Club Hipico Santiago & 306 & 24 & 8,5 \\
Hipódromo Chile Santiago & 236 & 16 & 6,8 \\
Valparaiso Sporting Club & 124 & 0 & 0,0 \\
Total & 666 & 40 & 6,0 \\
\hline
\end{tabular}


Fasciolosis en equina fina sangre de carrera - H. Alcaíno et al.

Tabla 3. Prevalencia de la infección por Fasciola hepatica en equinos F.S.C. de los hipódromos centrales de Chile y su distribución según sexo

\begin{tabular}{lccc}
\hline Sexo & $\begin{array}{c}\text { Caballos F.S.C } \\
\text { Examinados }\end{array}$ & \multicolumn{2}{c}{$\begin{array}{c}\text { Infectados con } \\
\text { Fasciola hepatica } \\
\text { n }\end{array}$} \\
\hline Hembras & 285 & 14 & \% \\
Machos & 381 & 26 & 4,9 \\
Total & 666 & 40 & 6,8 \\
\hline
\end{tabular}

Tabla 4. Prevalencia de la infección por Fasciola hepatica en equinos F.S.C. de los hipódromos centrales de Chile y su distribución según edad

\begin{tabular}{|c|c|c|c|}
\hline Edad & $\begin{array}{c}\text { Caballos } \\
\text { F.S.C }\end{array}$ & $\begin{array}{r}\text { In } \\
\text { Fasc }\end{array}$ & $\begin{array}{l}\text { s con } \\
\text { atica }\end{array}$ \\
\hline & Examinados & $\mathbf{n}$ & $\%$ \\
\hline 3 años o memores & 432 & 27 & 6,0 \\
\hline Mayores de 3 años & 214 & 13 & 6,1 \\
\hline Total & 666 & 40 & 6,0 \\
\hline
\end{tabular}

Tabla 5. Prevalencia de la infección por Fasciola hepatica en equinos F.S.C de los hipódromos centrales de Chile en los años 1982 y 2003

\begin{tabular}{lrrrrrr}
\hline Hipódromo/Ciudad & N* & $\begin{array}{r}\mathbf{1 9 8 3}^{\mathbf{7}} \\
\mathbf{n}^{* *}\end{array}$ & $\mathbf{\%}$ & $\mathbf{N}$ & $\begin{array}{r}\mathbf{2 0 0 3} \\
\mathbf{n}\end{array}$ & $\mathbf{\%}$ \\
\hline Santiago (HCH y CHS) & 955 & 100 & 10,5 & 542 & 40 & 7,4 \\
Viña del Mar(VSC) & 108 & 11 & 10,2 & 124 & 0 & 0,0 \\
Total & 1.063 & 111 & 10,4 & 666 & 40 & 6.0 \\
\hline
\end{tabular}

$\mathrm{N}^{*}=$ Número de F:S:C examinados

' $n * *=$ Número de F.S.C infectados con F.hepatica

\section{DISCUSIÓN}

El 6\% de infección encontrado en este estudio es mucho menor al $23,4 \%$ encontrado en F.S.C de la provincia de Nuble y ligeramente menor al $8,1 \%$ que se encontró en Linares ${ }^{4,5}$. También es inferior a los informes entregados por el Ministerio de Salud de Chile ${ }^{9}$, en donde figura una prevalencia de un $11 \%$ de infección por $F$. hepatica en caballos faenados en el período 19861987.

Sin embargo, resulta más interesante comparar los resultados obtenidos en el presente estudio con los encontrado hace 20 años atrás en caballos F.S.C radicados en los mismos hipódromos (Tabla 5) ${ }^{7}$. Esta comparación establece que la prevalencia global de infección por $F$. hepatica en los tres hipódromos, disminuyó significativamente desde un $10,4 \%$ hasta un $6 \%$ (p < $0,05)$.

También se produjo una disminución (Tabla 5) en los hipódromos de la Región Metropolitana (HCH y CHS) y en V Región (VSC) ( $<<0,05)$. Es así, como en el último de ellos (VSC) disminuyó del 10,2\% a $0 \%$ y en la Región Metropolitana si bien la disminución no fue tan manifiesta (del $10,5 \%$ al $7,4 \%$ ), también lo fue estadísticamente significativa. $(\mathrm{p}<0,05)$.
Creemos que disminución de la prevalencia de la infección por $F$. hepatica que se ha registrado en los hipódromos de la zona central del país corresponde a una expresión del efecto positivo que debe haber tenido en los criadores de caballos y los médicos veterinarios, los estudios que se realizaron hace dos décadas acerca de esta infección en los caballos y que despertó el interés por controlar esta enfermedad en esta especie, lo cual a esa fecha ignoraban que pudiese existir en tal magnitud.

Al analizar los resultados obtenidos en relación a la prevalencia de esta infección según sexo no se encontraron diferencias significativas $(\mathrm{p}>$ $0,05)$ lo que concuerda con otros trabajos ${ }^{6-8}$.

Respecto a la distribución de la infección según la edad no se determinaron diferencias significativas en los F.S.C (Tabla 4) lo que no concuerda con estudios anteriores ${ }^{4-8}$. También, el porcentaje de infección obtenido en los equinos F.S.C. de 3 años o menores es bastante más bajo que lo que encuentran esos autores. Esta disminución de las infecciones en los F.S:C en este grupo etáreo nos permite inferir que los profesionales están tomando en consideración la necesidad de controlar en forma temprana a $F$. hepatica de acuerdo a la conclusión de un trabajo anterior $^{7}$ que indicaba que la mayoría de los 
equinos F.S.C no adquieren la infección en el mismo hipódromo sino que vienen infectados desde su lugar de crianza. Con respecto a este antecedente, podemos informar que adicionalmente se averiguó el lugar de origen de los caballos que se encontraron infectados en el presente estudio y se pudo concluir que ninguno procedía de algún criadero que fue encontrado positivo en el estudio anterior ${ }^{7}$, lo que indicaría que los animales fueron tratados tempranamente.

A través de este estudio se ha intentado actualizar la información sobre la presentación de $F$. hepatica en equinos F.S.C. así como también se ha pretendido determinar, desde un punto de vista epidemiológico, el impacto de esta infección en los equinos que están participando actualmente en los hipódromos centrales del país. Tal impacto es menor al determinado por estudios anteriores, pero no deja de ser importante constatar que existe un $6 \%$ de caballos en competencia que aún están infectados, lo que nos debe motivar a continuar insistiendo en la implementación periódica de tratamientos antiparasitarios apropiados que les permita llegar en óptimas condiciones a las carreras en las que participan. Esta preocupación por los equinos F.S.C. de los hipódromos centrales debiera enfocarse a los hipódromos de provincias donde aún no existe una asesoría profesional concreta a los equinos F.S.C. que en ellos participan y, por ende, aún existen limitaciones para el desarrollo de la hípica nacional. Así queda planteada más que una inquietud, una necesidad de determinar el impacto epidemiológico y patológico no sólo de $F$. hepatica sino que de otros parásitos que afectan el rendimiento de los caballos.

\section{RESUMEN}

El propósito de este trabajo es entregar una mayor información sobre la situación actual de la fasciolosis en los caballos F.S.C. de los hipódromos centrales del país y evaluar indirectamente el funcionamiento de los esquemas sanitarios que se han implementado en estos últimos años.

Se recolectaron muestras de heces de caballos entre septiembre del año 2002 y junio del año 2003. Estas muestras fecales fueron obtenidas de 666 equinos F.S.C. radicados en los hipódromos de la $\mathrm{V}$ región y Región Metropolitana, y se obtuvieron de manera proporcional respecto a la población total de caballos existentes durantes el período de recolección.

Se encontró un $6 \%$ de los caballos F.S.C. muestreados positivos a la presencia de huevos de Fasciola hepatica ubicándose todos estos caballos en la Región Metropolitana y repartidos entre el Club Hípico de Santiago y el Hipódromo Chile. No hubo diferencias de infección con respecto a la edad y sexo. Con respecto a la tendencia de infección por $F$. hepatica de los caballos F S C se observa una disminución a través de los últimos 20 años $(\mathrm{p}<0,05)$.

\section{REFERENCIAS}

1.- BORAY J. Experimental fasciolasis in Australia. Adv. Parasitol 1969; 7: 96-204.

2.- MULLEN $P$ A. The diagnosis of liver disfunction in farm animals and horses. Vet Rec 1976; 99: 330-4.

3.- OWEN J. Liver fluke infection in horses and ponies. Equine Vet J 1977; 9: 29-31.

4.- SAN MARTIN C M. Frecuencia de distomatosis en Fina Sangre de Carrera y Chilenos en haras de la provincia de Nuble. Tesis Esc. Med Vet Universidad de Concepción 1979.

5.- MUÑOZ R L. Recuento de huevos de parásitos gastrointestinales y frecuencia de distomatosis en equinos fina sangre de carrera y chilenos en haras de la provincia de Linares. Tesis, Esc Med Vet Universidad de Concepción, Chile 1980.

6.- ALCAÍNO H, GORMAN T, GUEVARA G, FERNANDEZ J. D. Distomatosis y parásitos del intestino grueso de equinos de la zona centro sur de Chile. Arch Med Vet 1983; 15: 27-35.

7.- ALCAÍNO H, GORMAN T, PHILLIPS J. Distomatosis en equinos Fina Sangre de Carrera en Haras e Hipódromos de las regiones $\mathrm{V}$ y Metropolitana de Chile. Parasitol al Día 1983; 7: 37-40.

8.- ALCAINO H, VEGA F, KLEIN P, et al. Fascioliasis en caballos y conejos silvestres (Oryctolagus cuniculis) en la provincia de Curicó, Chile, Parasitol al Día 1988; 12: $136-40$

9.- CHILE. Ministerio de Salud. Información estadística de las principales enfermedades detectadas en los animales beneficiados en los mataderos del país durante el año 1987. Circular No 502. 1988.

10.- MORALES M A, LUENGO J, VASQUEZ J. Distribución y tendencia de la fasciolosis en ganado de abasto en Chile, 1989-1995. Parasitol al Día 2000; 24: 115-8.

11.- KISH L. Survey sampling. John Wiley and Sons, N Y, 643 pp 1965.

12.- TAGLE I. Enfermedades Parasitarias de los animales domésticos. Parte Primera. Editorial Andrés Bello, Santiago, Chile 1970 pp 193-209. 